Sousa, JC, Altino, IP, Andrade, SM, Cunha, MA, Araújo, BAC \& Oliveira, EH. (2020). Ketogenic diet for patients with type II diabetes mellitus. Research Society and Development. 9(7):1-20, e117973972.

\title{
Dieta cetogênica para pacientes com diabetes mellitus tipo II
}

Ketogenic diet for patients with type II diabetes mellitus

Dieta cetogénica para pacientes con diabetes mellitus tipo II

Recebido: 25/04/2020 | Revisado: 26/04/2020 | Aceito: 28/04/2020 | Publicado: 02/05/2020

Jailson Carmo de Sousa

ORCID: https://orcid.org/0000-0002-0196-0295

Faculdade Maurício de Nassau, Brasil

E-mail: jailson51462@gmail.com

Iranilsa Pereira Altino

ORCID: https://orcid.org/0000-0002-8973-4267

Faculdade Maurício de Nassau, Brasil

E-mail: iranilsaaltino@hotmail.com

Sâmia Moreira de Andrade

ORCID: https://orcid.org/0000-0002-2310-2515

Centro Universitário Santo Agostinho, Brasil

E-mail: samia.andrade27@hotmail.com

Maurício Almeida Cunha

ORCID: https://orcid.org/0000-0002-9752-5035

Faculdade Pitágoras, Brasil

E-mail: mauriciocferraz@ hotmail.com

Bismark Azevedo Cruz de Araújo

ORCID: https://orcid.org/0000-0001-8730-3132

Universidade Luterana do Brasil, Brasil

E-mail: bismarkazevedo@yahoo.com

Evaldo Hipólito de Oliveira

ORCID: https://orcid.org/0000-0003-4180-012X

Universidade Federal do Piauí, Brasil

E-mail: evaldohipolito@gmail.com 


\title{
Resumo
}

A diabetes mellitus tipo II em pleno século XXI é um dos maiores problema de saúde pública, que atinge grande parte da população e é a forma de maior predominância no mundo e correspondendo a cerca de $90 \%$ de todos os casos de diabetes, disfunção de uma epidemia crescente de pais desenvolvido e em desenvolvimento. Observou-se que a sua causa está relacionada principalmente com problemas de obesidade e que a perda de peso favorece ao tratamento. Com isso, a inclusão de uma dieta e a modificação no estilo vida pode contribuir de maneira positiva para uma melhora dos portadores dessa doença. A partir disso, o presente trabalho teve como objetivo realizar uma revisão integrativa sobre a influência da dieta cetogênica como auxilio no tratamento da diabetes mellitus tipo II e compreender tal manejo que possa contribuir para o individuo diabético. Trata-se de uma revisão integrativa, sendo utilizado como método de coleta de dados, com pesquisas em período publicado nas bases de dados: Scielo, Pubmed, Capes e órgão relevantes da área. Foram selecionados artigos de 2011 a Outubro de 2019 em português espanhol e inglês encontrados em resumos e textos completos. Por meio desse estudo conclui-se que a dieta cetogênica apresenta uma redução de massa corporal auxilia na redução da resistência a insulina melhora os níveis de glicose plasmática e consequentemente contribui para a redução da diabetes mellitus tipo II.

Palavras chaves: Diabetes Mellitus tipo II; Dieta cetogênica; Cetose e Insulina.

\begin{abstract}
Abastract
Type II diabetes mellitus in the 21st century is one of the biggest public health problems, which affects a large part of the population and is the most prevalent form in the world, corresponding to about $90 \%$ of all cases of diabetes, dysfunction of a growing epidemic of developed and developing parents. It was observed that its cause is mainly related to obesity problems and that weight loss favors treatment. Thus, the inclusion of a diet and the change in lifestyle can contribute positively to an improvement in patients with this disease. From this, the present study aimed to carry out an integrative review on the influence of the ketogenic diet as an aid in the treatment of type II diabetes mellitus and to understand such management that can contribute to the diabetic individual. It is an integrative review, being used as a method of data collection, with research in the period published in the databases: Scielo, Pubmed, Capes and relevant body in the area. Articles from 2011 to October 2019 were selected in Spanish and English Portuguese found in abstracts and full texts. Through this study it was concluded that the ketogenic diet has a reduction in body mass, helps to reduce
\end{abstract}


insulin resistance, improves plasma glucose levels and consequently contributes to the reduction of type II diabetes mellitus.

Key words: Diabetes Mellitus type II; Ketogenic diet; Ketosis and Insulin.

\section{Resumen}

La diabetes mellitus tipo II en el siglo XXI es uno de los mayores problemas de salud pública, que afecta a una gran parte de la población y es la forma más prevalente en el mundo, que corresponde a aproximadamente el $90 \%$ de todos los casos de diabetes, la disfunción de un creciente epidemia de padres desarrollados y en desarrollo. Se observó que su causa está relacionada principalmente con problemas de obesidad y que la pérdida de peso favorece el tratamiento. Por lo tanto, la inclusión de una dieta y el cambio en el estilo de vida pueden contribuir positivamente a una mejora en los pacientes con esta enfermedad. A partir de esto, el presente estudio tuvo como objetivo llevar a cabo una revisión integradora sobre la influencia de la dieta cetogénica como ayuda en el tratamiento de la diabetes mellitus tipo II y comprender el manejo que puede contribuir al individuo diabético. Es una revisión integradora, que se utiliza como método de recopilación de datos, con investigación en el período publicado en las bases de datos: Scielo, Pubmed, Capes y el organismo relevante en el área. Los artículos de 2011 a octubre de 2019 se seleccionaron en español e inglés y se encontraron en resúmenes y textos completos. A través de este estudio se concluyó que la dieta cetogénica tiene una reducción en la masa corporal, ayuda a reducir la resistencia a la insulina, mejora los niveles de glucosa en plasma y, en consecuencia, contribuye a la reducción de la diabetes mellitus tipo II.

Palabras clave: Diabetes mellitus tipo II; Dieta cetogénica; Cetosis e Insulina.

\section{Introdução}

O Diabetes mellitus (DM) é uma das doenças crônicas não transmissíveis (DCNT) ocupando a quarta principal causa de morte no mundo, em conjunto com a doença renal crônica estabelecem um impacto relevante nos sistema de saúde mundial e brasileiro (Ducan, 2014). Cerca de 463 milhões de individuo com diabetes no mundo só no Brasil 16,5 milhões de pessoas são portadoras dessa doença é o pais com maior numero de pessoas com diabetes da América Latina é o quinto país no mundo com pessoas com diabetes dados da IDF 2019, em 2017 cerca de 415 milhões de individuo eram portadores dessa patologia e estima-se para 2045 com a constante crescente da doença uma expectativa de mais de 700 milhões de 
portadores uma perspectiva de aumento de $51 \%$ de diagnósticos (International Diabetes Federation, 2019).

O DM como sendo um distúrbio metabólico caracteriza pelo o aumento da glicose plasmática (hiperglicemia) sendo resultante em defeito na secreção da insulina e na sua ação (Sociedade Brasileira de Diabetes, 2017). Nos diabetes tipo II eles produzem insulina mais suas células não conseguem utilizá-lo adequadamente por diminuição na ação (resistência insulínica), são dividida em dois tipos 1A e 1B, o tipo 1A (auto-imune) destruição das células beta pancreáticas e tipo 1B (idiopático) não tem causa estabelecida e corresponde aos casos onde não há presença de marcadores imunes (Moreira \& Carvalho, 2016).

Embora o diabetes mellitus tipo II seja uma distúrbio metabólico, estudos apontam como uma doença nutricional que a melhor estratégia para manter a saúde de forma equilibrada, os níveis de glicose plasmática e diminuir os riscos de complicações mais graves é mudanças no hábito alimentar (American Diabetes Association, 2017). A dieta cetogênica caracteriza-se por uma dieta rica em gorduras pobre em hidratos de carbono e com teor moderados de proteínas, vitaminas e minerais e como resultado a esse tipo de dieta são produzidos corpos cetônicos que são utilizados pelo corpo como a principal fonte de energia provinda através da lipólise e $\beta$-oxidação das gorduras, esse tipo de dieta por si só exige uma alteração complexa na alimentação e muitas vezes pode não ser tão bem toleradas pelo individuo no início mais é sim adaptável, pois o corpo passa por um processo de cura (Rola, 2014).

Com o aumento expressivo no diabete mellitus tipo II (DM2) decorrente da má qualidade ou deficiência alimentar, mudanças culturais, posição socioeconômica e geográfica e visto que a doença abrange de 85 a $90 \%$ do total de casos de diabetes, é relevante o uso de uma dieta com baixo níveis de carboidrato que diminui significativamente a HBalc (hemoglobina glicada) levando a perda de peso seja benéfica ao individuo pois a dieta é severamente restrita em carboidratos e tem como principal macronutrientes as gorduras benéficas, buscando produzir um estado de cetose constante no organismo que passa a utilizar as gorduras armazenadas que irá produzir corpos cetônicos para gerar energia (Silva et al., 2018).

A dieta tem como princípio a utilização de nutrientes pobres em carboidratos, baseando-se no fato de que, havendo uma grande restrição de carboidratos, vai levar a uma cetose, e, com isso, ocorrer uma oxidação lipídica, além de promover um efeito de saciedade e um aumento do gasto energético, fatores que devem promover um balanço energético negativo e consequente perda de peso, a denominação dessa dieta se deve ao fato de que, 
quando a gordura é consumida metabolicamente a mesma se decompõe em glicerol e ácidos graxos livres, dando origem a pares de dois compostos de carbono, denominados corpos cetônicos, no qual resulta um novo ácido graxo que será usado na forma de combustível energético (Bonnie et al., 2014).

Portanto baseando-se na deficiência alimentar e na carência de atividade física por parte do diabético, nota-se a importância do estudo da dieta cetogênica para pacientes com diabetes mellitus tipo II enfatizando o benefício da mesma para estes pacientes que poderão utiliza-lo como auxilio e tendo como resultados melhoras no quadro diabético. Com bases nessas informações, o objetivo desse trabalho é compreender a aplicação da dieta cetogênica em pessoas com diabetes mellitus tipo II, que poderão usar como forma de auxílio no seu tratamento.

\section{Metodologia}

Neste estudo foi realizado uma coleta de dados secundários através de levantamento bibliográfico, constituindo um tarbalho de revisão integrativa, onde nos artigos científicos para a elaboração desta revisão pesquisou-se em plataformas eletrônicas com busca de árticos pertinentes ao tema "Dieta cetogênica para pacientes com diabetes mellitus tipo II" Pereira, et al., 2018).

Para levantamento de dados realizou-se buscas nas bases Scielo-Ecientific Eletronic Library Onlyne, Pubmed-National Library of Medicine National Institutesof Health, Capescoordenação de aperfeiçoamento de pessoa de nível superior acadêmico e órgão importante na área, no recorte temporal de fevereiro de 2019 a Outubro 2019 utilizando as palavras chaves:

Diabetes, Diabetes Mellitus tipo II, Dieta cetogênica ou ketogenic diet, cetose e insulina com inserção de artigos originais a fim de se obter uma busca mais refinada e focando no assunto pertinente a revisão.

Os filtros utilizados na busca foram: artigos em inglês espanhol e português publicado entre 2011 e Outubro de 2019. Foram encontrados 627 artigos. O Quadro 1 a seguir mostra os artigos encontrados nas bases. 
Research, Society and Development, v. 9, n. 7, e117973972, 2020

(CC BY 4.0) | ISSN 2525-3409 | DOI: http://dx.doi.org/10.33448/rsd-v9i7.3972

Quadro 1. Numero de artigos recuperados segundos a base de dados

\begin{tabular}{|l|l|}
\hline Base de dados & Numero de artigos \\
\hline Scielo & 213 \\
\hline Pubmed & 231 \\
\hline CAPES & 183 \\
\hline Total & 627 \\
\hline
\end{tabular}

Fonte: Autores, 2019

A partir do banco de dados eletrônicos foram encontrados os seguintes artigos: 213 na base Scielo, 231 Pubmed e 183 na CAPES, totalizando 627 artigos para participarem da seleção e identificação dos artigos de interesse.

Para a identificação dos trabalhos de interesse para estudos foi realizada a leitura do título e dos resumos dos artigos encontrados, sendo este o critério de elegibilidade. Foram desconsiderados todos aqueles que não contemplavam a dieta cetogênica para diabetes mellitus tipo II.

Figura 1. Fluxograma, estratégia de busca de artigos

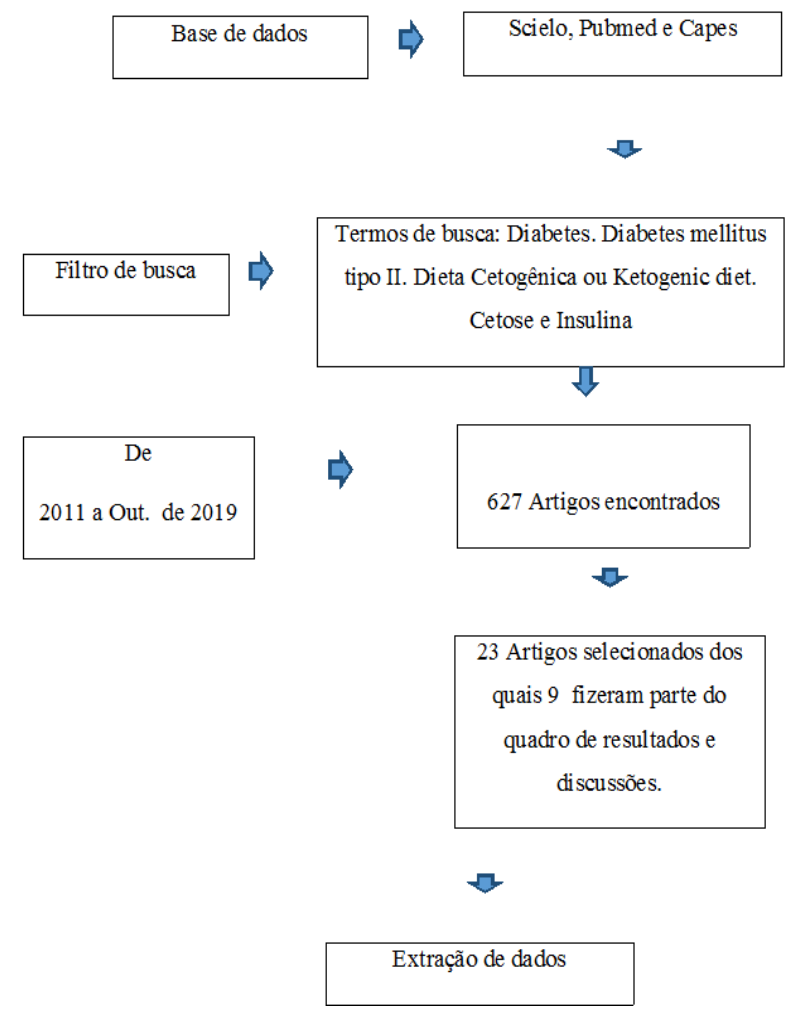

Fonte: Autores. 
Com a busca nas bases de dados supracitados, foram obtidos 627 artigos sobre o assunto. Após a leitura dos resumos e aplicação dos critérios de inclusão 23 artigos foram selecionados para esta revisão dos quais 9 foram selecionados para o quadro de resultados e discussão desta revisão integrativa.

Após realizar a leituras dos títulos e resumos dos 627 artigos acerca do tema 23 foram selecionados segundo os critérios estabelecidos, ou seja, deveriam tratar da dieta cetogênica em diabetes tipo II, da aplicabilidade da dieta nessa patologia além de estarem escrito em inglês espanhol e português.

Os artigos excluídos tinham como característica já serem uma revisão integrativa ou não possuíam versão online completa disponível, artigos que não foram encontrados na íntegra e cujas informações não estavam relacionadas ao foco da pesquisa.

\section{Resultados e Discussões}

De acordo com os estudos pré-estabelecidos, a dieta cetogênica caracterizada por uma dieta rica em gorduras, pobre em hidratos de carbono e com teor adequado de proteínas, vitaminas e minerais com recurso a esse tipo de dieta são produzidos corpos cetônicos que são utilizados como energia através da lipólise e $\beta$-oxidação (Rola, 2014 ).

A dieta cetogênica tem sido investigada no sentido de controlar os níveis de glicose no sangue e desta forma normalizar a sensibilidade à insulina, tem grande potencial clínico, contudo tendo em conta as limitações existentes na literatura, é necessário um estudo de mais casos e evidências a fim de conceitualizar a melhor abordagem para os pacientes destinados.

Antes do aparecimento da insulina exógena a modificação da dieta foi a principal terapia para diabetes. No entanto, as recomendações dietéticas durante esse tempo eram completamente diferentes das recomendações dietéticas atuais (Quadro 2) que consiste em baixo teor de gordura e alto índice de carboidratos para pacientes com diabetes (Hussain, 2012). 
(CC BY 4.0) | ISSN 2525-3409 | DOI: http://dx.doi.org/10.33448/rsd-v9i7.3972

Quadro 2: Atuais diretrizes dietéticas para portadores de DM:

\begin{tabular}{|l|l|}
\hline Macronutrientes & Ingestão Recomendada \\
\hline Valor energético total (VET) & $\begin{array}{l}\text { Considerar as necessidades individuas. } \\
\text { Utilizar parâmetro semelhantes a população } \\
\text { geral em todas as faixas etárias }\end{array}$ \\
\hline Carboidratos (CHO) & Carboidratos totais -45\%- 60\% \\
\hline Sacarose & $5 \%$ \\
\hline Frutose & Não se recomenda adição nos alimentos \\
\hline Fibras alimentar & $\begin{array}{l}\text { Mínimo de } 14 \mathrm{~g} / 1.000 \text { kcal } \\
\text { DM2 30 a } 50 \mathrm{~g} / \text { dia }\end{array}$ \\
\hline Gorduras totais (GT) & 20 a $35 \%$ VET \\
\hline Ácidos graxos saturados (AGS) & $<6 \%$ do VET \\
\hline Ácidos graxos poli-insaturados (AGPI) & Completar de forma individualizada \\
\hline Ácidos graxos monoinsaturados (AGMI) & 5 a $15 \%$ do VET \\
\hline Colesterol & $<300$ mg/dia \\
\hline Proteínas & 15 a $20 \%$ do VET \\
\hline
\end{tabular}

Fonte: sociedade brasileira de diabetes (2017-2018).

O Quadro 2, demonstra a quantidade recomendada de ingestão de macronutrientes por indivíduos portadores de DM. Essas recomendações são de acordo com Sociedade Brasileira de Diabetes. Portanto, vale ressaltar que a ingestão recomendada pode sofrer adequações de acordo com a necessidade do indivíduo.

As publicações descritas no Quadro 3, apresentam estudos que retratam os benéficos da dieta cetogênica no controle glicêmico, na melhora da tolerância a glicose, as possíveis alterações no metabolismo e os cuidados necessários para os indivíduos que seguem este tipo de dieta como tratamento alternativo da Diabetes Mellitus tipo II. Os resultados dos artigos, serão discutidos posteriormente. 
Quadro 3: Resultados dos artigos correlacionando Dieta Cetogênica e Diabetes Mellitus tipo II :

\begin{tabular}{|c|c|}
\hline Autor e ano de publicação & Resultado \\
\hline Freitas (2017) & $\begin{array}{l}\text { A dieta cetogênica parece melhorar o controle glicémico, } \\
\text { mas os doentes diabéticos que se encontrem fazendo uma } \\
\text { dieta cetogênica devem estar sob supervisão médica } \\
\text { rigorosa, porque esta dieta pode reduzir } \\
\text { significativamente os níveis de glicose no sangue } \\
\text { podendo agravar o quadro do indivíduo. }\end{array}$ \\
\hline Mencia, (2017) & $\begin{array}{l}\text { Dieta baixas em hidratos de carbono pode ser uma boa } \\
\text { alternativa para diabetes tipo II especialmente em pessoas } \\
\text { com sobre peso }\end{array}$ \\
\hline Hussain et al. (2012) & $\begin{array}{l}\text { Este estudo mostra os efeitos benéficos de uma dieta } \\
\text { cetogênica sobre o LCD convencional em obesos } \\
\text { diabéticos. A dieta cetogênica parece melhorar o controle } \\
\text { glicêmico. Portanto, pacientes diabéticos em uma dieta } \\
\text { cetogênica devem estar sob rigorosa supervisão médica } \\
\text { porque o LCKD pode reduzir significativamente os níveis } \\
\text { de glicose no sangue. }\end{array}$ \\
\hline Tay et al. (2014) & $\begin{array}{l}\text { A dieta cetogênica sustentou maiores reduções nas } \\
\text { necessidades de medicação para diabetes, e em melhorias } \\
\text { na estabilidade diurna da glicose no sangue e no perfil } \\
\text { lipídico do sangue, sugerindo uma maior otimização do } \\
\text { tratamento do DM2. }\end{array}$ \\
\hline Poplawski (2011) & $\begin{array}{l}\text { Este estudo demonstra que a Nefropatia diabética pode } \\
\text { ser revertida por uma dieta relativamente simples. Sendo } \\
\text { que a redução do metabolismo da glicose medeia os } \\
\text { efeitos protetores da dieta cetogênica portanto permanece } \\
\text { determinado }\end{array}$ \\
\hline \multirow[t]{2}{*}{$\begin{array}{l}\text { Pedersen et } \\
\text { al.(2014) }\end{array}$} & $\begin{array}{l}\text { Houve redução da hemoglobina glicada apenas } \\
\text { nos primeiros } 6 \text { meses, aos } 12 \text { meses houve redução } \\
\text { insignificante isso remete cautela ao uso a longo prazo. }\end{array}$ \\
\hline & Dietas baixas em Carboidratos como a cetogênica são \\
\hline
\end{tabular}


(CC BY 4.0) | ISSN 2525-3409 | DOI: http://dx.doi.org/10.33448/rsd-v9i7.3972

\begin{tabular}{|l|l|}
\hline Dyson (2015) & $\begin{array}{l}\text { eficazes para a melhora no controle glicêmico e } \\
\text { complicações cardiovasculares em diabéticos tipo 2. }\end{array}$ \\
\hline Zhang (2016) & $\begin{array}{l}\text { A dieta cetogênica melhora a tolerância à glicose e à } \\
\text { insulina em um modelo de diabetes em camundongos, } \\
\text { mas o acúmulo hepático de lipídios e a esteatose hepática } \\
\text { foram observados, o que deve ser considerado com } \\
\text { cautela na aplicação a longo prazo da dieta cetogênica. }\end{array}$ \\
\hline Emadian (2015) & $\begin{array}{l}\text { Qualquer dieta particular é superior no tratamento de } \\
\text { pacientes com sobrepeso e obesidade com DM2. Embora } \\
\text { a dieta mediterrânea, vegana e de baixo índice glicêmico } \\
\text { pareçam promissoras, pesquisas adicionais que controlam } \\
\text { a perda de peso e os efeitos dos medicamentos para } \\
\text { diabetes em amostras maiores são necessárias. }\end{array}$ \\
\hline
\end{tabular}

Fonte: Elaborado pelos autores

A dieta cetogênica é uma dieta que visa o emagrecimento e redução de massa corporal, onde, além de contribuir com a perda de peso, auxilia na diminuição da pressão arterial e da resistência à insulina, além de melhorar os níveis de glicose no sangue. Com isso, contribui para a redução da diabetes melittus tipo II. Freitas (2017) realizou um estudo em que os diabéticos passaram pela dieta cetogênica, porém, ao realizar esse tipo de dieta, os indivíduos devem estar sob rigorosa supervisão médica, pois a mesma pode reduzir significativamente os níveis de glicose no sangue dos pacientes que foram submetidos à longas datas. O estudo mostrou ainda que, mesmo sem significativa perca de peso, os pacientes da amostra reduziram os níveis de glicose sanguínea deixando claro que as dietas cetogênica mesmo sem diminuir o peso melhoram os níveis de glicose sanguínea.

Mencía (2017), afirma que dietas baixas em hidratos de carbono pode ser uma boa alternativa para diabetes tipo II especialmente em pessoas com sobre peso, a dieta tem demonstrado ser efetiva no controle glicêmico, pois obtém um bom resultado tanto na glicemia basal quanto na hemoglobina glicada de 7,3 caiu para uma média de 5,2 em 67\% dos participantes. Foi observado um resultado maior em curto prazo, porém é fundamental um estudo mais aprofundado pra estabelecer em longo prazo um resultado sólido, sendo que as mesmas tem a mesma quantidade de calorias. 
Em um estudo de Hussain (2012) feito com a duração de 23 semanas em adultos com índice de massa corporal (IMC) acima de $25 \mathrm{kmgm}^{2}$ foi realizado uma dieta restrita em carboidratos $20 \mathrm{~g} /$ dia, obtiveram um resultado que a dieta cetogênica teve efeito positivo sobre o peso corporal medida da cintura, triglicerídeos séricos e controle glicêmico em pacientes com diabetes tipo II, portanto concluíram que a dieta pobre em hidratos de carbono teve eficácia na redução da glicose plasmática no sangue porém é aconselhável que outros estudos seja feitos com mais pacientes até mesmo para examinar o ideal ajuste para medicamento antidiabético e agentes diuréticos para evitar as possíveis complicações de interações, hipoglicemia e desidratação devido a dieta, sendo necessário que indivíduo submetidos a dieta esteja em rigorosa supervisão médica ajustando a medicação conforme necessário para cada indivíduo.

Em Zhang (2016), observou que a dieta cetogênica vem sendo bastante utilizada para o controle de peso e glicemia, porém, notou-se que efeitos colaterais vêm surgindo, devido ao tratamento á longo prazo com a dieta cetogênica. Dessa forma, concluiu-se que a dieta cetogênica melhora a tolerância à glicose e à insulina em um modelo de diabetes em camundongos; no entanto, o acúmulo hepático de lipídios e a esteatose hepática foram observados, bem como alterações em gene chave importantes no metabolismo. Com isso, deve-se, de um modo geral, haver cautela na aplicação da dieta cetogênia em longo prazo pelo fato de problemas renais e outras complicações.

Poplawski (2011), em seu estudo, observou recentemente que o ácido cetona 3-betahidroxibutírico (3-OHB) reduz as respostas moleculares à glicose, e levantou a hipótese de que uma dieta cetogênica, que produz elevação prolongada de 3-OHB, pode reverter processos patológicos causados por diabetes. Estudos mostram que a cetona 3-betahidroxibutírico (3-OHB) bloqueia os efeitos moleculares da glicose e que a dieta cetogênica eleva os níveis de cetona 3-beta-hidroxibutirico (3-OHB) no sangue. Subsequentemente, ocorre a redução do metabolismo da glicose em alguns tecidos, incluindo o rim, e, com isso, há o favorecimento da reversão de neuropatia diabética, além de uma possível retinopatia.

Nos estudos de Hussain et al. (2012), Tay et al (2014) os resultados foram diferentes entre os grupos, o grupo de baixo carboidratos teve uma eficácia muito maior na redução dos níveis de glicose sanguínea, com relação ao outro grupo de estudo, que era composto de uma dieta de baixa caloria. Com relação à hemoglobina glicada, a redução também foi maior no grupo com restrição a carboidratos ao final das 24 semanas de estudo favorecendo então que a dieta cetogênica nesse estudo foi eficaz na redução dos níveis de glicose. 
De acordo com Dyson (2015), Pedersen (2014), os resultados também foram similares, em curto prazo, a dieta teve um grande efeito no controle glicêmico, perda de peso e controle de risco cardiovascular, porém em longo prazo não houve diferença significante em relação à outras intervenções. Portanto é necessário fazer um levantamento mais aprofundado com mais indivíduos principalmente em longo prazo para se tiver uma resposta mais precisa.

Emadian (2015) afirma que qualquer dieta particular se sobrepõe no tratamento de pacientes com sobrepeso e obesos portadores de Diabetes Mellitus tipo II. Embora outras dietas conhecidas, como a vegana e a de baixo índice glicêmico, sejam promissoras, não se apresentam eficientes na perca de peso. Em sua pesquisa de revisão, o autor, em uma revisão sistemática, obteve quatro estudos em que os efeitos significativos da dieta são questionáveis pelo fato de ter falha no controle das medicações e baixa adesão nas dietas prescritas, concluindo que ainda assim necessitam de pesquisas mais apuradas acerca do tema.

Inicialmente, nos anos 20 e 30, a dieta cetogênica era utilizada como uma estratégia terapêutica para pacientes com epilepsia, doença caracterizada por desarranjo do sistema nervoso central que acarretava com maior frequência em criança (Gomes et al., 2017).

A Dieta Cetogênica Clássica DC oferta em torno de $90 \%$ da sua energia na forma de gordura e $10 \%$ de carboidratos e proteínas uma característica típica da dieta cetogênica é sua habilidade em sintetizar uma grande quantidade de corpos cetônicos (cetogênese) e, assim aumentar de forma mensurável as concentrações circulantes e urinarias desses metabólitos fazendo com que o individuo que faz uso dessa dieta fique com hálitos cetônico e entre em estado de Cetose fisiologia normal da dieta cetogênica que é diferente de ceto-acidose principalmente no diabético, essa complicação acarreta mais os tipo I (Galan-Lopez et al., 2018).

A cetoacidose diabética (CAD) caracterizada como uma complicação aguda acometida no diabetes mellitus (DM) isso se dar ao aumento dos níveis de glicose no sangue tendo como relevância um estado de acidose metabólica desidratação e cetose, decorrente da deficiência profunda de insulina, acomete principalmente no diabetes mellitus tipo I e geralmente é precipitada devido a infecções ou até mesmo uso inadequado de insulina (omissão de administração de insulina) ou desconhecimento do diagnóstico de diabetes, a CAD pode ser a forma clinica inicial do DMT1 ou mesmo do tipo II é observado no diagnostico de DM1 de 5 a $45 \%$ dos pacientes submetidos a exames estima que a CAD tenha uma incidência anual de cerca de 1 a 5\% da população (Barone, at al., 2017).

$\mathrm{O}$ entendimento clinico da cetoacidose diabética CAD, preconiza-se o conhecimento da fisiopatologia dessa situação clinica que tem como deficiência absoluta a insulina 
geralmente esta associado ao aumento do hormônio glucagon que é um antagonista da insulina aumento dos glicocorticoides e catecolaminas, a hiperglicemia resulta tanto na diminuição da utilização periféricos de glicose como no aumento da sua produção endógena (glicogenólise e neoglicogênese) o desenvolvimento da cetoacidose é progressivo que passa de cetose inicial com acidose compensada até graus avançado de acidose metabólica e hipercetonemia com aparecimento de hálito cetônico (Galan-Lopez et al., 2018).

$\mathrm{Na}$ dieta cetogênica, a gordura é o nutriente em maior abundância e é através dela que vem a energia para os processos vitais. A gordura cai na circulação na forma de ácidos graxos (AGs) e segue até o fígado, onde será clivada pela $\beta$-oxidação formando acetil-CoA; essa, por sua vez, pode ser oxidada pelo ciclo de Krebs ou pode formar os corpos cetônicos.

Os corpos cetônicos produzidos no fígado têm como função servir como principal substrato energético para os outros tecidos, como, por exemplo, o coração, rins, cérebro e músculo esquelético em situações nas quais a oferta de glicose é baixa (Mencia, 2017).

A fim de produzir e manter um estado de cetose, a dieta cetogênica fornece uma alternativa energética para o cérebro em combinação com outro substrato que são as cetonas estimulando o organismo a entrar em estado de jejum de maneira que o jejum diminui a glicose o soro e resulta em menor proporção de insulina e glucagon, a diminuição dessa relação e o efeito na mudança em vários outros hormônios por exemplo adrenalina isso estimula a lipólise nos adipócitos no sangue, são liberados ácidos graxos que sofre oxidação através do fígado e músculos esquelético e cardíacos, com isso a oxidação desses ácidos graxos livre na mitocôndrias desses tecidos resulta na formação de acetil-coezima A (CoA) que condensa como oxalá-cetato para entrar no ciclo do acido tri-carboxílico com isso o fígado converte o acetil- CoA em acetoacetato (Galan-Lopez., 2018).

Existem diferentes tipos de DC descrito na literatura com o objetivo de aumentar a palatabilidade, e diminuir os efeitos secundários possibilitando a sua administração a um maior numero de individuo que esteja apto à dieta cetogênica e obter resultados com ela.

O Quadro 4 mostra a distribuição percentual dos macronutrientes contidos nas diferentes dietas encontradas na literatura que apresentam a mesma finalidade da cetogênica. Porém com distribuição dos macronutrientes em percentual e proporções diferentes, onde se adequam a finalidade e necessidade de cada dieta. 
(CC BY 4.0) | ISSN 2525-3409 | DOI: http://dx.doi.org/10.33448/rsd-v9i7.3972

Quadro 4- distribuição dos macronutrientes dos diferentes tipos de dieta com relação a dieta cetogênica.

\begin{tabular}{|l|l|l|l|l|}
\hline Tipo de Dieta & $\begin{array}{l}\text { Gordura } \\
(\% \text { VET })\end{array}$ & $\begin{array}{l}\text { Proteína } \\
(\% \text { VET })\end{array}$ & $\begin{array}{l}\text { HC } \\
(\% \text { VET })\end{array}$ & $\begin{array}{l}\text { Relação } \\
\text { Cetogênica }\end{array}$ \\
\hline DC clássica & $90 \%$ & $10 \%$ & $10 \%$ & $4: 1,3: 1$ \\
\hline DC com TCM & $\begin{array}{l}11 \% \quad+60 \% \\
\text { TCM }\end{array}$ & $10 \%$ & $19 \%$ & $1,2: 1$ \\
\hline $\begin{array}{l}\text { DC TCM } \\
\text { combinada }\end{array}$ & $\begin{array}{l}41 \%+30 \% \\
\text { TCM }\end{array}$ & $10 \%$ & $19 \%$ & $1,2: 1$ \\
\hline $\begin{array}{l}\text { Dieta Atkins } \\
\text { modificada }\end{array}$ & $60-70 \%$ & $20-30 \%$ & $6 \%$ & $1: 1$ \\
\hline $\begin{array}{l}\text { Dieta de baixo } \\
\text { índice } \\
\text { glicêmicio }\end{array}$ & $35-40 \%$ & $15-20 \%$ & IG<50 & \\
\hline
\end{tabular}

Fonte: Rola, 2014

VET: valor energético total; DC: Dieta cetogênica; TCM: triglicerídeo de cadeia média

Tradicionalmente na dieta cetogênica clássica DCC, $90 \%$ das necessidades energética diária provem das gorduras, garantindo que a obtenção de energia seja feita apartir do metabolismo dos lipídeos que são na maioria triglicerídeos de cadeia longa (TCL) enquanto que as restantes $10 \%$ provem dos $\mathrm{HC}$ e proteínas, calculadas através de uma relação entre a quantidade de gorduras em gramas e as de proteína e HC fornecidas em conjunto também em gramas, esta relação cetagênica poderá ser de: 3:1 ou 4:1 ou seja para cada 3 ou 4 gramas de gorduras se oferece 1 grama de proteína e HC conjuntamente (Rola, 2014). As modificações da dieta cetogênica podem ser feita em função de cada doente garantindo que não há complicações partindo das suas necessidades.

Não há nenhuma evidência sólida para apoiar o uso indiscriminado de dieta com $20 \mathrm{~g}$ ou menosde carboidratos por 90 dias principalmente em indivíduo com idade superior a 50 anos, há uma necessidade urgente por estudos mais longos e com maior numero de participantes com o objetivo de avaliar a eficácia da dieta em diabetes mellitus tipo II.

Rola, (2017) afirma que antes de iniciar a dieta cetogênica é de extrema importância a avaliação do doente para excluir a presença de qualquer patologia que seja contra indicado a DC para que possa excluir a existência de qualquer doença que vem a se agravar com a dieta. 
O Quadro 5 a seguir mostra as principais complicações para o uso da dieta cetogênica, onde é possível observar as principais complicações em decorrência do uso dieta cetogênica. Principalmente em indivíduos que apresentam fatores genéticos ou intrínsecos que predispõem tais complicações. Por este motivo o Quadro 5 apresenta as principais contraindicações relativa e absoluta de acordo com o fator individual.

Quadro 5: Contra indicação para o uso da dieta cetogênica

\begin{tabular}{|c|c|}
\hline Contra-indicação absolutas & $\begin{array}{l}\text { - Defeito na } \beta \text {-oxidacao } \\
\text { - Deficiência de desidrogenasedeacil- } \\
\text { CoA de cadeia média (MCAD) } \\
\text { - Deficiência de carboxilase do } \\
\text { piruvato }\end{array}$ \\
\hline Contra-indicação relativas & 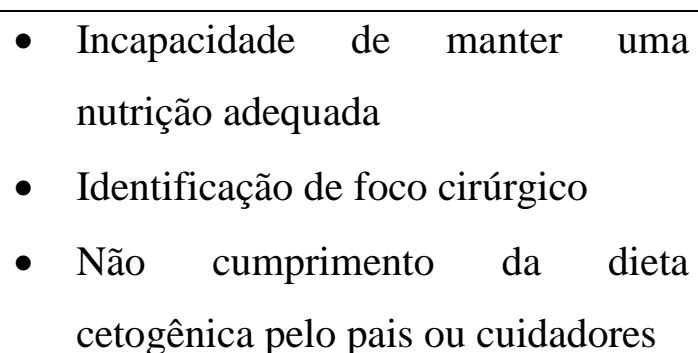 \\
\hline
\end{tabular}

Fonte: Rola, 2014

O controle glicêmico intensificado pode prevenir ou retardar o aparecimento das complicações crônicas nos diabetes mellitus e o carboidrato da dieta é o principal determinante da glicemia pos-prandial sendo o IG e a CG úteis para prever a resposta glicêmica (Silva et al., 2018).

A resistência insulínica (RI) acontece quando o organismo perde a capacidade de utilizar a glicose através da insulina, a célula beta pancreática aumenta a produção e secreção de insulina para compensar essa deficiência enquanto a tolerância à glicose permanece normal, a obesidade fator comum no diabetes mellitus tipo II está predominantemente associado à resistência a insulina principalmente do tipo centrípeto (abdominal) e da utilização periférica da glicose, a hiperinsulinemia que acompanha essa resistência leva a hipertensão arterial, dislipidemia que promove o desenvolvimento de aterosclerose (Fonseca, 2018). 
(CC BY 4.0) | ISSN 2525-3409 | DOI: http://dx.doi.org/10.33448/rsd-v9i7.3972

Uma variedade de fatores intrínsecos e extrínsecos pode determinar seu impacto na glicemia e influenciá-lo através da alimentação. O Quadro 6 mostra os fatores que podem influenciar e de que forma o índice glicêmico através da alimentação.

Quadro 6. Fatores que influenciam o índice glicêmico dos alimentos.

\begin{tabular}{|c|c|}
\hline Fatores & Influência sobre o IG dos alimentos \\
\hline Tipo de amido & $\begin{array}{ll} & \text { Razão amilose/amilopectina } \\
& \text { elevada (arroz parboilizado }\end{array}$ \\
\hline Monossacarídeos & - IG frutose $<$ IG glicose mel \\
\hline Acidez & - Retarda o esvaziamento gastrico \\
\hline Inibidores de $\alpha$-amilase & - Neveis elevado de lecitina \\
\hline
\end{tabular}

Fonte: Autores, 2019.

De acordo com os estudos analisados, inexiste na literatura uma definição consensual de dieta com teor baixo de carboidrato (Dieta cetogênica). Ainda que alguns autores sugiram utilizar parâmetros percentuais de calorias ingeridas na forma de carboidrato, a maioria utiliza o valor diário de 130g como limiar para definir a dieta. (Dyson, 2015; Noakes, 2016). No entanto, como existem evidências de que os benefícios da dieta podem ser maiores com restrições mais pronunciadas da ingesta de carboidratos, alguns autores têm sugerido utilizar o limiar de cetose como parâmetro adicional para caracterizar a esse tipo de dieta. Assim, caso a ingesta seja inferior a 50g/dia, estaria caracterizada a dieta com teor muito baixo de carboidratos. As definições mais aceita na literatura estão na Tabela 1 a seguir.

Tabela 1. Definições de dietas conforme o teor de carboidratos

\begin{tabular}{ll}
\hline Descrição da Dieta & $\begin{array}{l}\text { Quantidade de } \\
\text { carboidrato }\end{array}$ \\
\hline
\end{tabular}

\begin{tabular}{lll} 
Teor muito baixo de carboidrato & $20-50$ & 10 \\
\hline Teor baixo de carboidrato & $<130$ & $<26$ \\
\hline Teor moderado de carboidrato & $130-230$ & $26-45$ \\
\hline Teor elevado de carboidrato & $\mathbf{> 2 3 0}$ & $>\mathbf{4 5}$
\end{tabular}

Fonte: Dyson (2015); Noakes, (2016) Adaptado (2019). 
Não é possível definir um teor padrão de ingestão de carboidratos na dieta cetogência, pois a dieta deverá se adequar aos perfis individuais. Porém estudos demonstram que a baixa dieta em carboidratos se mostram eficazes na perda de peso e melhorias no controle glicêmico, em curto prazo. Disso isso, a dieta cetogênica deve seguir as necessidades de cada indivíduo e sempre se atentar as contraindicações de pessoas que apresentam fatores que predispõem possíveis complicações associadas ao uso da dieta.

\section{Considerações Finais}

O estudo se demonstra relevante para a comunidade científica e indivíduos que pretendem aderir à dieta cetogênica. Pois irá contribuir proporcionando entendimento sobre as vertentes que ainda são pertinentes em torno do uso. Demostrando os benefícios, contraindicações e possíveis complicações.

Como mostra no estudo, é evidente o quão positiva é a perda de peso através da dieta cetogênica, para o auxílio na melhoria do quadro de diabetes mellitus tipo II. Contudo, durante o estudo, concluímos que a dieta cetogênica é a melhor dieta para ser seguida pelos portadores de diabetes mellitus tipo II, pois além de possibilitar uma perda de peso mais rápida e redução de massa corporal, a mesma auxilia na diminuição da pressão arterial e da resistência à insulina, onde diminui o índice de problemas cardiovasculares, melhora os níveis de glicose no sangue e consequentemente contribui para a redução da diabetes mellitus tipo II.

Os estudos demonstraram que não há um limiar padrão alimentar para ingestão de carboidratos ou de outros macronutrientes (proteínas e gorduras). Além do risco eminente de possíveis complicações associadas ao uso da dieta cetogênica. Dessa forma, até o momento algumas informações importantes são inconclusivas. Então sugere-se para trabalhos futuros, o aprofundamento e estudos mais detalhados sobre essas informações já supracitadas, para sanar quaisquer dúvidas.

\section{Referências}

American Diabetes Association. (2017). Standards of medical care in diabetes, 40(1): 33-40.

Barone, B. et al0: (2017). Cetose diabetic em adultos - atualização de uma complicação antiga. Arq. Bras. Endocrinol. Metab. Rio de janeiro, 51(9): 1434-1447. 
Duncan, B. B. et al. (2017). The burdem of diabetes and hyperglycemia in brasil and its states: finding from the global burden of disease study 2015. Rev. bras. Epidemol. São Paulo 20(1): 90-101.

Dyson, P. (2015). Low carbohydrate diets and type 2 diabetes: what is the latest evidence? Diabetes Ther, 6(4): 411-424.

Emadian, A., Andrews, R. C. et al. (2015). The effect of macronutrients on glycaemic control: a systematic review of dietary randomised controlled trials in overweight and obese adults with type 2 diabetes in which there was no difference in weight loss between treatment groups. British Journalof Nutrition. 114(10): 1656-1666.

Fonseca, E. J. N .C. et al. (2018). Síndrome Metabólica e Resistência Insulínica pelo HomaIR no Climatério. International Journal of Cardiovascular Sciences. 31(3)201-208.

Freitas, C. M. S. V. S. (2017). Dieta cetogênica no tratamento da obesidade.. 22 f. Monografia (Mestrado em Nutrição) - Instituição académica: Faculdade de Ciências da Nutrição e Alimentação da Universidade do Porto, Porto.

Hussain, T. A et al, (2012). Effect of low-calorie low carboidrateketogenic diet in type 2 diabetes. Rev. Nutrition. 28(10): 1016-21.

International Diabetes Federation, (2019). IDF Diabetes Atlas, Ninth edition.

Johnstone, A. M., et al. (2008). Effects of a high-protein ketogenic diet on hunger, appetite, and weight loss in obese men feeding ad libitum. The American Journal of Clinical Nutrition. Bethesda-Md, 87(1): 44-55.

Galan-Lopez, P., Ries, F., Gisladottir, T., Domínguez, R., Sánchez-Oliver, A.J. (2018). Healthy lifestyle: Relationship between mediterranean diet, body composition and physical fitness in 13 to 16 year old icelandic students. Int. J. Environ. Res. Public Health. v. 2632, 2018, p.1-15. 
Mencía, J, V, et al. (2017). Dietas bajas en hidratos de carbono para diabéticos de tipo 2. Revisión sistemática. Nutrición Hospitalaria, 34(1): 224-234.

Moreira, R. A. S., (2016). Treinamento resistido e seus benefícios em relação ao diabetes mellitus tipo 1: relato de experiência. Trabalho de Conclusão de Curso. Departamento de Educação Física do Centro de Ciências Biológicas e da Saúde da Universidade Federal da Paraíba. Campina Grande - PB.

Noakes, T. D., Windt, J. (2016). Evidence that supports the prescription of lowcarbohydratehigh-fat diets: a narrative review. British Journal of Sports Medicine, 51(2): 133-139

Paoli, A. et al. (2013). Long Term Successful Weight Loss with a Combination Biphasic Ketogenic Mediterranean Diet and Mediterranean Diet Maintenance Protocol. Nutrients, Basel-Switzerland, 5(12): 5205-5217.

Pedersen, E. et al. (2014). High protein weight loss diets in obese subjects with type 2 diabetes mellitus. Nutrition, Metabolism and Cardiovascular Diseases, 24(5):554-62.

Pereira, A.S. et al. (2018). Metodologia da pesquisa científica. [e-book]. Santa Maria. Ed. UAB/NTE/UFSM. Disponível em: https://repositorio.ufsm.br/bitstream/handle/1/15824/Lic_Computacao_MetodologiaPesquisa-Cientifica.pdf?sequence=1. Acesso em: 26 Abril 2020.

Poplawski, M. M. et al. (2011). Reversal of diabetic nephropathy by a ketogenic diet. PLoSOne, 6(4): e18604.

Rola, M., Vasconcelos, C. (2014). Dieta Cetogénica-Abordagem Nutricional. Rev.nutricias, 22: $16-19$.

Silva, M. F., Steemburgo, T., Azevedo, J. M., Mello, D. V. (2018). Papel do índice glicêmico e da carga glicêmica na prevenção e no controle metabólico de pacientes com diabetes mellitus tipo 2, Arquivos Brasileiros de Endocrinologia \& Metabologia, 53(5): 560-571. 
Sociedade Brasileira de Diabetes. (2017). Diretrizes da sociedade brasileira de diabetes 2017-2018. São Paulo. Sociedadebrasileira de diabetes.12p.

Tay, J., Marsh, N. D. L., Thompsom, C. H., Noakes, M., Buckley, D. J., Witter, G. A., Yancy, W. S. JR., Brinkworth, D. G. (2014). A very low-carbohidrate, low-saturated fat diet for type 2 diabetes management: Rev. A randomized trial. Nutr. 37(11): 2909-18.

Tomé, A., Amorim, S. T. S. P., Mendonça, D. R. B. (2003). Dieta cetogênica no tratamento das epilepsias graves da infância: percepção das mães. Revista de Nutrição, Campinas, 16(2): 203-210.

Zhang, X. et al. (2016). Long-term ketogenic diet contributes to glycemic control but promotes lipid accumulation and hepatic steatosis in type 2 diabetic mice. Nutrition research, 36(4): 349-358.

\title{
Porcentagem de contribuição de cada autor no manuscrito
}

\author{
Jailson Carmo de Sousa - 20\% \\ Iranilsa Pereira Altino - 20\% \\ Sâmia Moreira de Andrade - 15\% \\ Maurício Almeida Cunha - 15\% \\ Bismark Azevedo Cruz de Araújo - 15\% \\ Evaldo Hipólito de Oliveira - 15\%
}

